\title{
Unbounded normal derivative for the Stokes system near boundary
}

\author{
Kyungkeun Kang
}

\begin{abstract}
We study local boundary regularity for the Stokes system. We show that, unlike in the interior case, non-local effects can lead to a violation of local regularity in the spatial variables near the boundary.
\end{abstract}

\section{Introduction}

In this paper we study local boundary regularity for the Stokes system. Let $B_{1}^{+}=$ $\left\{x \in \mathbb{R}^{3}:|x|<1, x_{3}>0\right\}, \Lambda^{\prime}=\partial B_{1}^{+} \cap\left\{x_{3}=0\right\}$, and $\Lambda_{+}=\partial B_{1}^{+} \cap\left\{x_{3}>0\right\}$. We consider the system:

$$
\left.\begin{array}{c}
u_{t}-\Delta u+\nabla p=0 \\
\operatorname{div} \mathrm{u}=0
\end{array}\right\} \text { in } B_{1}^{+} \times(0, T)
$$

where $T>0$. We will assume $u=0$ on $\Lambda^{\prime} \times(0, T)$ and $u=0$ when $t=0$. We emphasize that homogeneous boundary condition is assigned only on the flat part of the boundary of $B_{1}^{+}$. We do not impose restrictions on $u$ at $\Lambda_{+} \times(0, T)$.

If we consider a similar situation for the heat equation, $u_{t}-\Delta u=0$, we have the classical local boundary regularity result that

$$
\sup _{Q_{\frac{r}{2}}^{+}}\left|D_{x}^{l} D_{t}^{m} u\right| \leq C_{l, m, r, n}|u|_{L^{2}\left(Q_{r}^{+}\right)},
$$

where $Q_{r}^{+} \subset B_{1}^{+} \times(0, T)$ is a parabolic cylinder and $C$ depends on $l, m, r$ and $n$, but not on $u$. Because of non-local effect of the pressure, estimates of this form cannot be expected to hold for system (1). Nevertheless, G. Seregin recently in [9] proved the following result for (1) under above assumptions:

$$
[u]_{\mathcal{C}_{\text {Par }}^{\alpha}\left(Q_{\frac{r}{2}}^{+}\right)} \leq C_{r, n, \alpha}\left(|\nabla u|_{L^{2}\left(Q_{r}^{+}\right)}+|p|_{L^{\frac{3}{2}}\left(Q_{r}^{+}\right)}\right)
$$

where $0<\alpha<\frac{2}{3}$ and $C$ depends on $r, n$, and $\alpha$, but not on $u$ (see Lemma 2 in [9, page 219]).

In this paper I construct examples showing that the result in [9] cannot be extended to the derivative $D_{x_{3}} u$. Roughly speaking, we can have $\sup \left|\mathrm{D}_{\mathrm{x}_{3}} \mathrm{u}\right|=\infty$ while the right-hand side of (2) is finite. We conjecture that similar phenomenon occurs for 
the Navier-Stokes equations. (A construction of such singular solution of the NavierStokes equations would not solve the main regularity problem for these equations, since in our case the appearance of the singularity is caused by non-local behavior of the solutions and boundary conditions.) In a way, our solutions are a boundary analogue of the well-known solution of the form $u(x, t)=\nabla h(x, t)$ where $h(x, t)$ is harmonic with respect to the spatial variable (see [10]), which are relevant for questions regarding interior regularity. However, non-trivial solutions of the form $u(x, t)=\nabla h(x, t)$ with $\left.u\right|_{\Lambda^{\prime}}=0$ do not exist at the boundary.

The plan of this paper is as follows:

In Section 2, we review the representation formula for the Stokes system in a half space in [12] and we set up our example.

In Section 3, after basic observations, we show that the solution we construct is bounded in a region considered.

In Section 4, we obtain a priori estimate of the normal derivative and show that normal derivatives of the solution we construct are unbounded near the boundary at a certain time.

In Section 5, we show that constructed solution is indeed a weak solution and the pressure associated with it is in a reasonable Lebesgue space. At the end of this section, we briefly explain another example, which is again a weak solution but Hölder continuity fails at the boundary.

\section{Preliminaries}

In this section we collect notation and definitions used throughout this paper. We shall also recall results in [12] for the Stokes system in a half space in $\mathbb{R}^{3}$.

- We denote by $\mathbb{R}_{+}^{3}$ as a half-space of $\mathbb{R}^{3}$. For $x \in \mathbb{R}^{3}$ and $r>0$ we denote by $B_{x, r}$ the open ball $\left\{y \in \mathbb{R}^{3}:|x-y|<r\right\}$. If $r=1$, we write $B_{x, 1}\left(\right.$ or $B_{x, 1}^{+}$) as $B_{x}$ (or $B_{x}^{+}$) for simplicity. We also denote by $D_{1}$ the unit disk in $\mathbb{R}^{2}$, i.e. $D_{1}=\left\{x^{\prime} \in \mathbb{R}^{2}:\left|x^{\prime}\right|<1\right\}$.

- Let $x, y \in \mathbb{R}^{n}$ where $n=2,3$. We denote the distance between $x$ and $y$ by $d(x, y)$. If there is no confusion, we sometimes denote $d(x, y)$ by $\bar{d}$. Let $A \subset \mathbb{R}^{n}$ and $x \in \mathbb{R}^{n}$. Then $d(x, A)$ indicates the distance between $x$ and $A$, i.e. $d(x, A)=\inf \{d(x, y): y \in$ $A\}$. Similarly, we write $d(A, B)$ as the distance between two sets $A$ and $B$.

- Let $\Omega \subset \mathbb{R}^{3}$ be a domain. For $1 \leq q \leq \infty, W^{k, q}(\Omega)$ denote the usual Sobolev space, i.e. $W^{k, q}(\Omega)=\left\{u \in L^{q}(\Omega): D^{\alpha} u \in L^{q}(\Omega), 0 \leq|\alpha| \leq k\right\}$. As usual, $W_{0}^{k, q}(\Omega)$ is the completion of $\mathcal{C}_{0}^{\infty}(\Omega)$ in $W^{k, q}(\Omega)$. We also denote by $\mathcal{C}^{\alpha}(\Omega)$ the space of Hölder continuous functions with the exponent $\alpha \in(0,1)$.

- Let $1 \leq q, r \leq \infty$ and $I=(0, T) . L^{r}\left(I ; W^{k, q}(\Omega)\right)$ is the Banach space consisting of all measurable functions with a finite norm

$$
\|u\|_{L^{r}\left(I ; W^{k, q}(\Omega)\right)}=\left(\int_{I}\|u(\cdot, t)\|_{W^{k, q}(\Omega)}^{r} \mathrm{~d} t\right)^{\frac{1}{r}} .
$$

If $q=r$ and $k=0$, then we simply write $L^{q}\left(I ; L^{q}(\Omega)\right)=L^{q}(\Omega \times I)$. We also denote 
by $\mathcal{C}_{x, t}^{\alpha, \frac{\alpha}{2}}(\Omega \times I)$ the space of Hölder continuous functions with the exponent $\alpha \in(0,1)$ from $\Omega \times I$ into $\mathbb{R}^{3}$. In the above definition, $\Omega \times I$ is endowed with a parabolic metric $d\left(z, z^{\prime}\right)=\left|x-x^{\prime}\right|+\left|t-t^{\prime}\right|^{\frac{1}{2}}$ where $z=(x, t)$ and $z^{\prime}=\left(x^{\prime}, t^{\prime}\right)$.

- Let $f: \mathbb{R}^{3} \times(0, T) \rightarrow \mathbb{R}^{3}, g: \mathbb{R}_{+}^{3} \rightarrow \mathbb{R}^{3}$ and $h: \mathbb{R}^{2} \times(0, T) \rightarrow \mathbb{R}^{2}$ be vector valued functions. Then $f *_{1} h$ and $g *_{2} h$ are defined as follows:

$$
\begin{gathered}
f *_{1} h(x, t)=\int_{0}^{t} \int_{\mathbb{R}^{2}} f\left(x-y^{\prime}, t-s\right) \cdot h\left(y^{\prime}, s\right) \mathrm{d} y^{\prime} \mathrm{d} s \\
g *_{2} h(x, t)=\int_{\mathbb{R}^{2}} g\left(x-y^{\prime}\right) \cdot h\left(y^{\prime}, t\right) \mathrm{d} y^{\prime} .
\end{gathered}
$$

- For a given scalar function $f$, we denote $f^{+}(x)=\max \{f(x), 0\}$ and $f^{-}(x)=$ $\max \{-f(x), 0\}$.

- We let $C$ denote the generic constant which may vary from line to line.

For simplicity, we denote $I=(0, T)$. We consider the following Stokes system:

$$
\begin{array}{cc}
u_{t}-\Delta u+\nabla p=0 \\
\operatorname{div} \mathrm{u}=0 \\
u=0 & \text { in } \mathbb{R}_{+}^{3} \times I \\
& \text { at } t=0
\end{array}
$$

with a given non-zero boundary condition:

$$
u\left(x^{\prime}, 0, t\right)=\left(0,0, v\left(x^{\prime}, t\right)\right) \text { on } \partial \mathbb{R}_{+}^{3} \times I
$$

where $v$ is a scalar function compactly supported in $D_{1} \times I \subset \mathbb{R}^{2} \times I$, which we will specify below.

Definition of boundary data $v \quad$ Let $s$ be a fixed positive time with $0<s<T$. We define $v: D_{1} \times I \rightarrow \mathbb{R}$ as follows:

$$
v\left(x^{\prime}, t\right)=g\left(\left|x^{\prime}\right|\right) h(t) \text { in } D_{1} \times I
$$

where $g$ and $h$ satisfy the following properties, respectively:

[S] $g$ is compactly supported, smooth, positive, radially symmetric and $g^{\prime}(\cdot)<0$ in $D_{1}$.

[T] $h$ is of the form

$$
h(t)=\left\{\begin{array}{cc}
(s-t)^{\frac{1}{2}} \xi(t) & \text { when } 0 \leq t<s \\
0 & \text { when } s \leq t<T
\end{array}\right.
$$

where $\xi$ is a cut off function satisfying $\xi \in \mathcal{C}_{0}^{\infty}(\mathbb{R}), 0 \leq \xi(x) \leq 1, \xi$ is compactly supported in $\left(\frac{s}{2}, \frac{3 s}{2}\right)$, and $\xi=1$ in $\left(\frac{3 s}{4}, \frac{5 s}{4}\right)$. We note that $h$ is Hölder continuous, but not Lipschitz continuous. 
By recalling the representation formula of the Stokes system in [12], we can have a solution $u$ of (3) with boundary data (4) where $v$ is given in (5) and $g$ and $h$ satisfy [S] and [T]. Before we represent the solution explicitly, we review some estimates of functions which will be used later (see [12, page 37, 41]). To avoid confusion, we follow notation used in [12].

$$
\begin{gathered}
\left|D_{x}^{l} D_{t}^{m} A(x, t)\right| \leq \frac{C}{t^{m+\frac{1}{2}}\left(x^{2}+t\right)^{\frac{l+1}{2}}} \\
\left|D_{x^{\prime}}^{l} D_{x_{3}}^{k} D_{t}^{m} B(x, t)\right| \leq \frac{C}{\left(x_{3}^{2}+t\right)^{m+\frac{k+1}{2}}\left(x^{2}+t\right)^{\frac{l+1}{2}}} \\
\left|D_{x^{\prime}}^{l} D_{x_{3}}^{k} D_{t}^{m} \mathcal{C}_{i}(x, t)\right| \leq \frac{C}{t^{m+\frac{1}{2}}\left(x^{2}+t\right)^{\frac{l+2}{2}}\left(x_{3}^{2}+t\right)^{\frac{k}{2}}},
\end{gathered}
$$

where $A, B$ and $\mathcal{C}_{i}$ are defined as follows (see [12, page 37, 40]).

$$
\begin{gathered}
A(x, t)=\left(\frac{1}{|x|} *_{2} \Gamma\left(x^{\prime}, 0, t\right)\right)=\int_{\mathbb{R}^{2}} \frac{\Gamma\left(y^{\prime}, 0, t\right)}{\left|x-y^{\prime}\right|} \mathrm{d} y^{\prime} \\
B(x, t)=\left(\frac{1}{\left|x^{\prime}\right|} * \Gamma\left(x^{\prime}, t\right)\right)=\int_{\mathbb{R}^{2}} \frac{\Gamma\left(x-y^{\prime}, t\right)}{\left|y^{\prime}\right|} \mathrm{d} y^{\prime} \\
\mathcal{C}_{i}(x, t)=\int_{\mathbb{R}_{2}} \mathrm{~d} y^{\prime} \int_{0}^{x_{3}} \frac{\partial \Gamma(y, t)}{\partial y_{3}} \frac{y_{i}-x_{i}}{|y-x|^{3}} \mathrm{~d} y_{3},
\end{gathered}
$$

where $\Gamma$ is the fundamental solution of the heat equation in $\mathbb{R}^{3}$. In addition, we recall relations between $B, \mathcal{C}_{i}$ and $\Gamma$ (see [12, page 40]).

$$
\frac{\partial \mathcal{C}_{\alpha}}{\partial x_{3}}=\frac{\partial \mathcal{C}_{3}}{\partial x_{\alpha}}+\frac{\partial^{2} B}{\partial x_{\alpha} \partial x_{3}} \text { where } \alpha=1,2, \quad \sum_{i=1}^{3} \frac{\partial \mathcal{C}_{i}}{\partial x_{i}}=-2 \pi \frac{\partial \Gamma}{\partial x_{3}} .
$$

Since the tangential components of boundary data are zero at the boundary (see (4)), in order to find the explicit representation of $u$, it suffices to recall the Green tensor $G_{i j}$ for $i=1,2,3$, and $j=3$, which are given as follows (see [12, page 36]):

$$
\begin{gathered}
G_{i 3}(x, t)=-2 \delta_{i 3} \frac{\partial \Gamma(x, t)}{\partial x_{3}}-\frac{1}{\pi} \frac{\partial}{\partial x_{3}} \int_{\mathbb{R}_{2}} \mathrm{~d} y^{\prime} \int_{0}^{x_{3}} \frac{\partial \Gamma(y, t)}{\partial y_{3}} \frac{y_{i}-x_{i}}{|y-x|^{3}} \mathrm{~d} y_{3} \\
-\frac{1}{2 \pi} \delta(t) \frac{\partial}{\partial x_{i}} \frac{1}{|x|} \quad \text { for } i=1,2,3 .
\end{gathered}
$$

Using the Green tensors above, the each component $u_{i}, i=1,2,3$ of velocity field of the Stokes system (3) with (4) is expressed as follows (see [12, page 53]):

$$
u_{i}=\left(G_{i 3} *_{1} v\right)=-2\left(\delta_{i 3} \frac{\partial \Gamma(x, t)}{\partial x_{3}} *_{1} v\right)-\frac{1}{\pi}\left(\frac{\partial \mathcal{C}_{i}}{\partial x_{3}} *_{1} v\right)-\frac{1}{2 \pi}\left(\frac{\partial}{\partial x_{i}} \frac{1}{|x|} *_{2} v\right)
$$

More specifically,

$$
u_{1}=-\frac{1}{\pi}\left(\frac{\partial \mathcal{C}_{1}}{\partial x_{3}} *_{1} v\right)-\frac{1}{2 \pi}\left(\frac{\partial}{\partial x_{1}} \frac{1}{|x|} *_{2} v\right)
$$




$$
\begin{gathered}
u_{2}=-\frac{1}{\pi}\left(\frac{\partial \mathcal{C}_{2}}{\partial x_{3}} *_{1} v\right)-\frac{1}{2 \pi}\left(\frac{\partial}{\partial x_{2}} \frac{1}{|x|} *_{2} v\right), \\
u_{3}=-2\left(\frac{\partial \Gamma(x, t)}{\partial x_{3}} *_{1} v\right)-\frac{1}{\pi}\left(\frac{\partial \mathcal{C}_{3}}{\partial x_{3}} *_{1} v\right)-\frac{1}{2 \pi}\left(\frac{\partial}{\partial x_{3}} \frac{1}{|x|} *_{2} v\right) .
\end{gathered}
$$

Finally, we conclude this section by reminding the representation formula of the pressure associated with $u$ (see [12, page 52]).

$$
\begin{gathered}
p(x, t)=\frac{1}{2 \pi} \frac{\partial^{2}}{\partial^{2} x_{3}}\left(\frac{1}{|x|} *_{2} v\right)-\frac{1}{\pi} \frac{\partial}{\partial x_{3}}\left(\frac{\partial}{\partial t}-\sum_{k=1}^{2} \frac{\partial^{2}}{\partial x_{k}^{2}}\right)\left(A *_{1} v\right) \\
+\frac{1}{2 \pi}\left(\frac{1}{|x|} *_{2} \frac{\partial v}{\partial t}\right)
\end{gathered}
$$

where $A$ is defined in (10).

\section{Basic Lemmas and bounded velocity}

Let us choose a point $x_{0}^{\prime} \equiv\left(x_{1}, 0,0\right) \in \partial \mathbb{R}_{+}^{3}$ sufficiently away from origin and $x_{1}>0$ (for instance we may take $x_{1}>10$ such that $\left(B_{x_{0}^{\prime}} \cap\left\{x_{3}=0\right\}\right) \cap D_{1}=\emptyset$ ). We denote $\Lambda^{\prime}=B_{x_{0}^{\prime}} \cap\left\{x_{3}=0\right\}$. Suppose that $u=\left(u_{1}, u_{2}, u_{3}\right)$ and $p$ are given in (12) and (13) where $v$ is given by (5) and $g$ and $h$ satisfy [S] and [T], respectively. From the representation formula (12) and (13), we easily see that $u, p \in L^{1}\left(\mathbb{R}_{+}^{3} \times I\right)$, and thus they are well-defined almost everywhere in $\mathbb{R}_{+}^{3} \times I$ and solve the Stokes system (3) in a distribution sense (see e.g. [6, page 87]) in $\mathbb{R}_{+}^{3} \times I$ (This can be done by regularizing $h$ and passing to the limit). In addition, we will show that $u$ is bounded in $B_{x_{0}^{\prime}}^{+} \times I$ (see Lemma 3.3) and $u$ and $p$ solve the following Stokes system in a weak sense (see Lemma 5.1 and Lemma 5.2)

$$
\left.\begin{array}{c}
u_{t}-\Delta u+\nabla p=0 \\
\operatorname{div} u=0
\end{array}\right\} \text { in } B_{x_{0}^{\prime}}^{+} \times I
$$

and $u$ satisfies initial and boundary conditions

$$
u=0 \text { at } B_{x_{0}^{\prime}}^{+} \times\{t=0\}, \quad u=0 \text { on } \Lambda^{\prime} \times I .
$$

It should be mentioned that $u$ satisfies zero boundary conditions in a point-wise sense on $\Lambda^{\prime}$ because $u$ is Hölder continuous up to the boundary (see Remark 4.2). Our main goal is to show that the normal derivatives of tangential components of $u$ are unbounded near $\Lambda^{\prime}$ when $t=s$, which will be shown in Main Lemma in section 4.

In this section, we show that velocity field $u$ is uniformly bounded by $L^{\infty}$-norm of $v$ and $D v$ for each $i=1,2,3$ in $B_{x_{0}^{\prime}}^{+} \times I$ where $D$ means the first order spatial derivative, not including time derivative. We shall start with simple observations for our proof. We consider 


$$
B\left(x-y^{\prime}, \tau\right)=\int_{\mathbb{R}^{2}} \frac{\Gamma\left(x-y^{\prime}-z^{\prime}, \tau\right)}{\left|z^{\prime}\right|} \mathrm{d} z^{\prime}=\frac{e^{-\frac{x_{3}^{2}}{4 \tau}}}{(4 \pi \tau)^{\frac{3}{2}}} K\left(x^{\prime}-y^{\prime}, \tau\right),
$$

where $K\left(x^{\prime}-y^{\prime}, \tau\right)$ is defined by

$$
K\left(x^{\prime}-y^{\prime}, \tau\right)=\int_{\mathbb{R}^{2}} \frac{e^{-\frac{\left|x^{\prime}-y^{\prime}-z^{\prime}\right|^{2}}{4 \tau}}}{\left|z^{\prime}\right|} \mathrm{d} z^{\prime} .
$$

Since $y^{\prime} \in D_{1}$ and $x^{\prime} \in \Gamma^{\prime}$, the distance $\left|x^{\prime}-y^{\prime}\right|$ is strictly positive. We note first that $K\left(x^{\prime}-y^{\prime}, \tau\right)$ holds the following scaling properties:

$$
\begin{aligned}
& K\left(\mu x^{\prime}-\mu y^{\prime}, \mu^{2} \tau\right)=\mu K\left(x^{\prime}-y^{\prime}, \tau\right) \\
& K\left(\frac{x^{\prime}}{\sqrt{\tau}}-\frac{y^{\prime}}{\sqrt{\tau}}, 1\right)=\frac{1}{\sqrt{\tau}} K\left(x^{\prime}-y^{\prime}, \tau\right) .
\end{aligned}
$$

Indeed, changing the variable $z^{\prime}=\mu z^{\prime}$, we have (18). The second one (19) is obtained by replacing $\mu$ by $\frac{1}{\sqrt{\tau}}$. In following Lemmas we calculate the upper bound and lower bound of $K\left(x^{\prime}-y^{\prime}, \tau\right)$.

Lemma 3.1 Let $x^{\prime} \in \Lambda^{\prime}, y^{\prime} \in D_{1}$, and $K$ be defined in (17). Then

$$
K\left(x^{\prime}-y^{\prime}, \tau\right) \leq \frac{8 \pi \tau}{\left|x^{\prime}-y^{\prime}\right|}+\pi\left|x^{\prime}-y^{\prime}\right| e^{-\frac{\left|x^{\prime}-y^{\prime}\right|^{2}}{16 \tau}} .
$$

Proof. With the aid of relations (18) and (19), it suffices to consider the case of $\tau=1$. For simplicity, we denote $\bar{d}=\left|x^{\prime}-y^{\prime}\right|$ and introduce three disjoint sets

$$
\begin{gathered}
\mathcal{A}=\left\{z^{\prime} \in \mathbb{R}^{2}:\left|x^{\prime}-y^{\prime}-z^{\prime}\right| \leq \frac{\bar{d}}{2}\right\} \\
\mathcal{B}=\left\{z^{\prime} \in \mathbb{R}^{2}:\left|z^{\prime}\right| \leq \frac{\bar{d}}{2}\right\}, \quad \mathcal{C}=\mathbb{R}^{2} \backslash(A \cup B) .
\end{gathered}
$$

Dividing integrand on each disjoint sets, we have

$$
\int_{\mathbb{R}^{2}} \frac{e^{-\frac{\left|x^{\prime}-y^{\prime}-z^{\prime}\right|^{2}}{4}}}{\left|z^{\prime}\right|} \mathrm{d} z^{\prime}=\int_{\mathcal{A}} \cdot+\int_{\mathcal{B}} \cdot+\int_{\mathcal{C}} \cdot \equiv I+I I+I I I .
$$

Consider the first term $I$. Using $\frac{1}{2} \bar{d} \leq\left|z^{\prime}\right| \leq \frac{3}{2} \bar{d}$ in $\mathcal{A}$, we have

$$
\begin{aligned}
I & =\int_{\mathcal{A}} \frac{e^{-\frac{\left|x^{\prime}-y^{\prime}-z^{\prime}\right|^{2}}{4}}}{\left|z^{\prime}\right|} \mathrm{d} z^{\prime} \leq \frac{2}{\bar{d}} \int_{\mathcal{A}} e^{-\frac{\left|x^{\prime}-y^{\prime}-z^{\prime}\right|^{2}}{4}} \mathrm{~d} z^{\prime} \\
& \leq \frac{4 \pi}{\bar{d}} \int_{0}^{\frac{\bar{d}}{2}} e^{-\frac{s^{2}}{4}} s \mathrm{~d} s \leq \frac{8 \pi}{\bar{d}}\left(1-e^{-\frac{\bar{d}^{2}}{16}}\right) .
\end{aligned}
$$

Since $\left|z^{\prime}\right| \leq \frac{1}{2} \bar{d}$ in $\mathcal{B}$, we obtain

$$
I I=\int_{\mathcal{B}} \frac{e^{-\frac{\left|x^{\prime}-y^{\prime}-z^{\prime}\right|^{2}}{4}} \mathrm{~d} z^{\prime}}{\left|z^{\prime}\right|} \leq \int_{\mathcal{B}} \frac{e^{-\frac{\bar{d}^{2}}{16}} \mathrm{~d} z^{\prime}}{\left|z^{\prime}\right|} \leq e^{-\frac{\bar{d}^{2}}{16}} \int_{\mathcal{B}} \frac{\mathrm{d} z^{\prime}}{\left|z^{\prime}\right|} \leq \pi \bar{d} e^{-\frac{\bar{d}^{2}}{16}} .
$$


Finally, similarly, the third term $I I I$ is estimated as follows.

$$
\begin{aligned}
I I I & =\int_{\mathcal{C}} \frac{e^{-\frac{\left|x^{\prime}-y^{\prime}-z^{\prime}\right|^{2}}{4}}}{\left|z^{\prime}\right|} \mathrm{d} z^{\prime} \leq \frac{2}{\bar{d}} \int_{\mathcal{C}} e^{-\frac{\left|x^{\prime}-y^{\prime}-z^{\prime}\right|^{2}}{4}} \mathrm{~d} z^{\prime} \\
& \leq \frac{4 \pi}{\bar{d}} \int_{\frac{\bar{d}}{2}}^{\infty} e^{-\frac{s^{2}}{4}} s \mathrm{~d} s \leq \frac{8 \pi}{\bar{d}} e^{-\frac{\bar{d}^{2}}{16}}
\end{aligned}
$$

To sum up, we get

$$
K\left(x^{\prime}-y^{\prime}, 1\right)=\int_{\mathbb{R}^{2}} \frac{e^{-\frac{\left|x^{\prime}-y^{\prime}-z^{\prime}\right|^{2}}{4}}}{\left|z^{\prime}\right|} \mathrm{d} z^{\prime} \leq \frac{8 \pi}{\bar{d}}+\pi \bar{d} e^{-\frac{\bar{d}^{2}}{16}}
$$

The scaling property (19) completes our proof.

Following similar procedures of the previous Lemma, we also have the lower bound of $K\left(x^{\prime}-y^{\prime}, \tau\right)$.

Lemma 3.2 Let $x^{\prime} \in \Lambda^{\prime}, y^{\prime} \in D_{1}$, and $K$ be defined in (17). Then

$$
K\left(x^{\prime}-y^{\prime}, \tau\right) \geq \frac{8 \pi \tau}{3\left|x^{\prime}-y^{\prime}\right|}\left(1-e^{-\frac{\left|x^{\prime}-y^{\prime}\right|^{2}}{16 \tau}}\right) .
$$

Proof. As the previous case, it suffices to consider the case of $\tau=1$. It follows the simple modification of the first term. Using $\frac{1}{2} \bar{d} \leq\left|z^{\prime}\right| \leq \frac{3}{2} \bar{d}$ on $\mathcal{A}$, we get

$$
\begin{aligned}
K\left(x^{\prime}-y^{\prime}, 1\right) & \geq \int_{\mathcal{A}} \frac{e^{-\frac{\left|x^{\prime}-y^{\prime}-z^{\prime}\right|^{2}}{4}}}{\left|z^{\prime}\right|} \mathrm{d} z^{\prime} \geq \frac{2}{3 \bar{d}} \int_{\mathcal{A}} e^{-\frac{\left|x^{\prime}-y^{\prime}-z^{\prime}\right|^{2}}{4}} \mathrm{~d} z^{\prime} \\
& =\frac{4 \pi}{3 \bar{d}} \int_{0}^{\frac{\bar{d}}{2}} e^{-\frac{s^{2}}{4}} s \mathrm{~d} s=\frac{8 \pi}{3 \bar{d}}\left(1-e^{-\frac{\bar{d}^{2}}{16}}\right) .
\end{aligned}
$$

The scaling property (19) completes our proof.

Using estimates of the above Lemmas, we can prove that $L^{\infty}$-norm of $u$ is estimated in terms $L^{\infty}$-norm of $v$ and its spatial derivative $D v$.

Lemma 3.3 Let $v$ be a function given in (5), and assume that $g$ and $h$ satisfy [S] and [T], respectively. Let $u$ be given by the representation formula (12). Then $u$ is bounded in $B_{x_{0}^{\prime}}^{+} \times I$ and the following estimate holds:

$$
\sup _{B_{x_{0}^{\prime}}^{+} \times I}|u| \leq C \sup _{D_{1} \times I}(|v|+|D v|)
$$

where $C=C\left(d\left(x_{0}^{\prime}, D_{1}\right), T\right)$.

Proof. For convenience, we denote $\tilde{d} \equiv d\left(x_{0}^{\prime}, D_{1}\right)$. We note first that there exists a positive constant $C>0$ such that $C^{-1} d\left(y, D_{1}\right)<\tilde{d}<C d\left(y, D_{1}\right)$ for all $y \in B_{x_{0}^{\prime}}^{+}$, because $x_{0}^{\prime}$ is sufficiently away from the support of $v$. Recalling the representation 
formula (12), let us consider first component $u^{1}$ of $u=\left(u^{1}, u^{2}, u^{3}\right)$ and write it, for convenience, as

$$
u_{1}(x, t) \equiv \tilde{u}(x, t)+\hat{u}(x, t)
$$

where

$$
\tilde{u}(x, t)=-\frac{1}{\pi}\left(\frac{\partial \mathcal{C}_{1}}{\partial x_{3}} *_{1} v\right)=-\frac{1}{\pi} \int_{\mathbb{R}^{2}} \mathrm{~d} y^{\prime} \int_{0}^{t} \frac{\partial \mathcal{C}_{1}\left(x-y^{\prime}, \tau\right)}{\partial x_{3}} v\left(y^{\prime}, t-\tau\right) \mathrm{d} \tau
$$

and

$$
\hat{u}(x, t)=-\frac{1}{2 \pi}\left(\frac{\partial}{\partial x_{1}} \frac{1}{|x|} *_{2} v\right)=-\frac{1}{2 \pi} \int_{\mathbb{R}^{2}} v\left(y^{\prime}, t\right) \frac{\partial}{\partial x_{1}} \frac{1}{\left|x-y^{\prime}\right|} \mathrm{d} y^{\prime} .
$$

Since $v$ is supported in $D_{1} \subset R^{2}$, the second term is bounded. Indeed,

$$
\begin{aligned}
|\hat{u}(x, t)| & \leq C \int_{D_{1}} \mathrm{~d} y^{\prime} \int_{0}^{t}\left|v\left(y^{\prime}, t\right) \frac{1}{\left|x-y^{\prime}\right|^{2}}\right| \mathrm{d} \tau \\
& \leq C \sup _{D_{1} \times I}|v| \frac{t}{d^{2}} \leq C \sup _{D_{1} \times I}|v| .
\end{aligned}
$$

Secondly, utilizing the relation (11), we rewrite $v(x, t)$ as follows,

$$
\begin{aligned}
\tilde{u}(x, t)= & -\frac{1}{\pi}\left(\frac{\partial \mathcal{C}_{1}}{\partial x_{3}} *_{1} v\right)=-\frac{1}{\pi}\left(\frac{\partial \mathcal{C}_{3}}{\partial x_{1}}+\frac{\partial^{2} B}{\partial x_{1} \partial x_{3}}\right) *_{1} v \\
= & -\frac{1}{\pi} \int_{\mathbb{R}^{2}} \mathrm{~d} y^{\prime} \int_{0}^{t} \mathcal{C}_{3}\left(x-y^{\prime}, \tau\right) \frac{\partial v\left(y^{\prime}, t-\tau\right)}{\partial y_{1}} \mathrm{~d} \tau \\
& -\frac{1}{\pi} \int_{\mathbb{R}^{2}} \mathrm{~d} y^{\prime} \int_{0}^{t} \frac{\partial B\left(x-y^{\prime}, \tau\right)}{\partial x_{3}} \frac{v\left(y^{\prime}, t-\tau\right)}{\partial y_{1}} \mathrm{~d} \tau \\
\equiv & \tilde{u}_{1}(x, t)+\tilde{u}_{2}(x, t) .
\end{aligned}
$$

With the aid of the estimate (9), $\tilde{u}_{1}$ can be controlled in terms of $|D v|$. Indeed,

$$
\begin{gathered}
\left|\tilde{u}_{1}(x, t)\right| \leq C\left|\int_{\mathbb{R}^{2}} \mathrm{~d} y^{\prime} \int_{0}^{t} \mathcal{C}_{3}\left(x-y^{\prime}, \tau\right) \frac{\partial v\left(y^{\prime}, t-\tau\right)}{\partial y_{1}} \mathrm{~d} \tau\right| \\
\leq C \sup _{D_{1} \times I}|D v| \int_{D_{1}} \mathrm{~d} y^{\prime} \int_{0}^{t} \frac{1}{\tau^{\frac{1}{2}}\left(\left(x-y^{\prime}\right)^{2}+\tau\right)} \mathrm{d} \tau \\
\leq C \sup _{D_{1} \times I}|D v| \frac{1}{\tilde{d}^{2}} \int_{0}^{t} \frac{\mathrm{d} \tau}{\tau^{\frac{1}{2}}} \leq C \sup _{D_{1} \times I}|D v| .
\end{gathered}
$$

It remains to estimate $\tilde{u}_{2}$. Using the estimate (20) of $K\left(x^{\prime}-y^{\prime}, \tau\right)$, we obtain

$$
\left|\tilde{u}_{2}(x, t)\right| \leq C \sup _{D_{1} \times I}|D v| \int_{D_{1}} \mathrm{~d} y^{\prime} \int_{0}^{t}\left|\frac{\partial B\left(x-y^{\prime}, \tau\right)}{\partial x_{3}}\right| \mathrm{d} \tau
$$




$$
\begin{gathered}
\leq C \sup _{D_{1} \times I}|D v| \int_{D_{1}} \mathrm{~d} y^{\prime} \int_{0}^{t} e^{-\frac{x_{3}^{2}}{4 \tau}} \frac{x_{3}}{\tau} \frac{K\left(x^{\prime}-y^{\prime}, \tau\right)}{\tau^{\frac{3}{2}}} \mathrm{~d} \tau \\
\leq C \sup _{D_{1} \times I}|D v| \int_{0}^{t} e^{-\frac{x_{3}^{2}}{4 \tau}} \frac{x_{3}}{\tau} \frac{1}{\tau^{\frac{3}{2}}} \frac{\tau}{d\left(x^{\prime}, y^{\prime}\right)} \mathrm{d} \tau \\
+C \sup _{D_{1} \times I}|D v| \int_{0}^{t} e^{-\frac{x_{3}^{2}}{4 \tau}} \frac{x_{3}}{\tau} \frac{1}{\tau^{\frac{3}{2}}} d\left(x^{\prime}, y^{\prime}\right) e^{-C \frac{d\left(x^{\prime}, y^{\prime}\right)^{2}}{4 \tau}} \mathrm{d} \tau \equiv I+I I .
\end{gathered}
$$

We can easily check that integral part of $I I$ is bounded, and thus we skip the details. For the first term $I$, using the change of variable $s=\frac{x_{3}}{\sqrt{4 \tau}}$, we have

$$
I \leq C \sup _{D_{1} \times I}|D v| \int_{\frac{x_{3}}{2 \sqrt{t}}}^{\infty} e^{-s^{2}} \mathrm{~d} s \leq C \sup _{D_{1} \times I}|D v| .
$$

This completes the assertion (22) for $u_{1}$. Following similar procedures, we obtain the same estimate for $u_{2}$. Next consider the third component $u_{3}$.

$$
u_{3}=-2\left(\frac{\partial \Gamma(x, t)}{\partial x_{3}} *_{1} v\right)-\frac{1}{\pi}\left(\frac{\partial \mathcal{C}_{3}}{\partial x_{3}} *_{1} v\right)-\frac{1}{2 \pi}\left(\frac{\partial}{\partial x_{3}} \frac{1}{|x|} *_{2} v\right) .
$$

First consider the last term.

$$
\left|\left(\frac{\partial}{\partial x_{3}} \frac{1}{|x|} *_{2} v\right)\right| \leq C \sup _{D_{1} \times I}|v| \int_{D_{1}} \frac{\partial}{\partial x_{3}} \frac{1}{\left|x-y^{\prime}\right|} \mathrm{d} y^{\prime} \leq C \sup _{D_{1} \times I}|v| \frac{1}{\tilde{d}^{2}} .
$$

Secondly, the first term is estimated as follows. We note first that

$$
\int_{D_{1}} e^{-\frac{\left|x^{\prime}-y^{\prime}\right|^{2}}{4 \tau}} \frac{1}{2 \tau} \mathrm{d} y^{\prime} \leq C,
$$

where $C$ is independent of $x^{\prime}$ and $\tau$. Indeed, the crucial fact is that $\left|x^{\prime}-y^{\prime}\right|$ never vanishes. With the aid of the scaling method, (26) can be easily verified. Using the estimate (26), we have

$$
\begin{gathered}
\left|\frac{\partial \Gamma(x, t)}{\partial x_{3}} *_{1} v\right| \leq C \int_{0}^{t} \int_{D_{1}}\left|e^{-\frac{\left|x^{\prime}-y^{\prime}\right|^{2}}{4 \tau}} v\left(y^{\prime}, t-\tau\right) \mathrm{d} y^{\prime} \frac{x_{3}}{2 \tau} \frac{e^{-\frac{x_{3}^{2}}{4 \tau}}}{(4 \tau)^{\frac{3}{2}}}\right| \mathrm{d} \tau \\
=C \sup _{D_{1} \times I}|v| \int_{0}^{t} \int_{D_{1}} e^{-\frac{\left|x^{\prime}-y^{\prime}\right|^{2}}{4 \tau}} \frac{1}{2 \tau} \mathrm{d} y^{\prime} x_{3} \frac{e^{-\frac{x_{3}^{2}}{4 \tau}}}{(4 \tau)^{\frac{3}{2}}} \mathrm{~d} \tau \\
\leq C \sup _{D_{1} \times I}|v| \int_{0}^{t} x_{3} \frac{e^{-\frac{x_{3}^{2}}{4 \tau}}}{(4 \tau)^{\frac{3}{2}}} \mathrm{~d} \tau \leq C \sup _{D_{1} \times I}|v| .
\end{gathered}
$$

Finally, using the relation (11) and the estimate (27), the second term in (25) can be estimated by the similar computations we did before, and thus we omit details. This completes the proof. 


\section{Normal derivatives near boundary}

In this section, we study a priori estimate of normal derivatives of velocity field $u$ represented in (12) in $B_{x_{0}^{\prime}}^{+} \times I$ of the Stokes system (3) with boundary data (4) where $v$ is given in (5) and $g$ and $h$ satisfies [S] and [T], respectively. Next, we prove that normal derivatives of $u$ blow up near $\Lambda^{\prime} \equiv B_{x_{0}^{\prime}} \cap\left\{x_{3}=0\right\}$ when $t=s$.

\subsection{A priori estimate}

In next Lemma, we assume that $v$ given in (4) is smooth in space and time variables.

Lemma 4.1 Let $v$ be a function given in (5), and assume that $g$ and $h$ are smooth and compactly supported in $D_{1}$ and $I$, respectively. Let $u$ be given by the representation formula (12). Then the normal derivative of $u$ is bounded in $B_{x_{0}^{\prime}}^{+} \times I$ and the following estimate holds:

$$
\sup _{B_{x_{0}^{\prime}}^{+} \times I}\left|D_{x_{3}} u\right| \leq C \sup _{D_{1} \times I}\left(|D v|+\left|D^{2} v\right|+\left|D_{t} v\right|\right)
$$

where $C=C\left(d\left(x_{0}^{\prime}, D\right), T\right)$.

Proof. Note first that it suffices to consider $D_{x_{3}} u_{i}, i=1,2$, because $D_{x_{3}} u_{3}$ is controlled by tangential derivatives due to $\operatorname{div} \mathrm{u}=0$. In addition, we estimate only $D_{x_{3}} u_{1}$. Since it follows similar procedures, we omit the detail for $D_{x_{3}} u_{2}$. Recalling representation formula, we denote $u_{1}(x, t) \equiv \tilde{u}(x, t)+\hat{u}(x, t)$ as the proof of Lemma 3.3 (see (23)). We note first that $\left|D_{x_{3}} \hat{u}\right| \leq C \sup _{D_{1} \times I}|D v|$. Indeed, since $v$ is supported in $D_{1}$, we have

$$
\left|2 \pi \frac{\partial \hat{u}(x, t)}{\partial x_{3}}\right| \leq \int_{D_{1}}\left|\frac{\partial}{\partial y_{1}} v\left(y^{\prime}, t\right) \frac{\partial}{\partial x_{3}} \frac{1}{\left|x-y^{\prime}\right|}\right| \mathrm{d} y^{\prime} \leq C \sup _{D_{1} \times I}|D v| .
$$

Therefore, it remains to analyze $D_{x_{3}} \tilde{u}$. As (24), we can express $\tilde{u}=\tilde{u}_{1}+\tilde{u}_{2}$. First, we consider the second term, $D_{x_{3}} \tilde{u}_{2}(x, t)$. Using $\left(\partial_{t}-\Delta_{x}\right) B\left(x-y^{\prime}, \tau\right)=0$, the integration by parts, and the estimate (8) of $B\left(x-y^{\prime}, \tau\right)$, we obtain

$$
\begin{gathered}
\left|\pi \frac{\partial \tilde{u}_{2}(x, t)}{\partial x_{3}}\right|=\left|\int_{\mathbb{R}^{2}} \mathrm{~d} y^{\prime} \int_{0}^{t} \frac{\partial^{2} B\left(x-y^{\prime}, \tau\right)}{\partial^{2} x_{3}} \frac{\partial v\left(y^{\prime}, t-\tau\right)}{\partial y_{1}} \mathrm{~d} \tau\right| \\
\leq \int_{\mathbb{R}^{2}} \mathrm{~d} y^{\prime} \int_{0}^{t} \sum_{i=1}^{2}\left|\frac{\partial B\left(x-y^{\prime}, \tau\right)}{\partial y_{i}} \frac{\partial^{2} v\left(y^{\prime}, t-\tau\right)}{\partial y_{1} y_{i}}\right| d \tau \\
\quad+\int_{\mathbb{R}^{2}} \mathrm{~d} y^{\prime} \int_{0}^{t}\left|\frac{\partial B\left(x-y^{\prime}, \tau\right)}{\partial y_{1}} \frac{\partial v\left(y^{\prime}, t-\tau\right)}{\partial \tau}\right| \mathrm{d} \tau \\
\leq C\left(\sup _{D_{1} \times I}\left|D^{2} v\right|+\sup _{D_{1} \times I}\left|D_{t} v\right|\right) .
\end{gathered}
$$


Consider the first term, $\tilde{u}_{1}(x, t)$. Taking derivative with respect to $x_{3}$ and using the relation (11), we obtain

$$
\begin{aligned}
& \left|\pi \frac{\partial \tilde{u}_{1}(x, t)}{\partial x_{3}}\right|=\left|\int_{\mathbb{R}^{2}} \mathrm{~d} y^{\prime} \int_{0}^{t} \frac{\partial \mathcal{C}_{3}\left(x-y^{\prime}, \tau\right)}{\partial x_{3}} \frac{\partial v\left(y^{\prime}, t-\tau\right)}{\partial y_{1}} \mathrm{~d} \tau\right| \\
& \quad \leq\left|\int_{\mathbb{R}^{2}} \mathrm{~d} y^{\prime} \int_{0}^{t} \sum_{i=1}^{2} \mathcal{C}_{i}\left(x-y^{\prime}, \tau\right) \frac{\partial^{2} v\left(y^{\prime}, t-\tau\right)}{\partial y_{1} y_{i}} \mathrm{~d} \tau\right| \\
& +2 \pi\left|\int_{\mathbb{R}^{2}} \mathrm{~d} y^{\prime} \int_{0}^{t} \frac{\partial \Gamma}{\partial x_{3}}\left(x-y^{\prime}, \tau\right) \frac{\partial v\left(y^{\prime}, t-\tau\right)}{\partial y_{1}} \mathrm{~d} \tau\right| \equiv I+I I .
\end{aligned}
$$

With the aid of (9), $I$ is estimated as follows:

$$
I \leq \int_{\mathbb{R}^{2}} \mathrm{~d} y^{\prime} \int_{0}^{t} \sum_{i=1}^{2}\left|\mathcal{C}_{i}\left(x-y^{\prime}, \tau\right) \frac{\partial^{2} v\left(y^{\prime}, t-\tau\right)}{\partial y_{1} y_{i}}\right| \mathrm{d} \tau \leq C \sup _{D_{1} \times I}\left|D^{2} v\right| .
$$

Following the similar computation as (27), we have

$$
I I \leq \int_{\mathbb{R}^{2}} \mathrm{~d} y^{\prime} \int_{0}^{t}\left|\frac{\partial \Gamma}{\partial x_{3}}\left(x-y^{\prime}, \tau\right) \frac{\partial v\left(y^{\prime}, t-\tau\right)}{\partial y_{1}}\right| \mathrm{d} \tau \leq C \sup _{D_{1} \times I}|D v| .
$$

To sum up, we get

$$
\left|\frac{\partial \tilde{u}(x, t)}{\partial x_{3}}\right| \leq C \sup _{D_{1} \times I}\left(|D v|+\left|D^{2} v\right|+\left|D_{t} v\right|\right) .
$$

This completes the proof.

\subsection{Unbounded normal derivative}

In this subsection, we estimate normal derivative of $u$ in $B_{x_{0}^{\prime}}^{+} \times I$. According to a priori estimate (28), we observe that every other terms except for $D_{x_{3}} \tilde{u}_{2}$ (see (29)) are controlled by spatial derivatives of $v$. Therefore, it suffices only to analyze $D_{x_{3}} \tilde{u}_{2}$. We first recall $D_{x_{3}} \tilde{u}_{2}$ in (29):

$$
\pi \frac{\partial \tilde{u}_{2}(x, t)}{\partial x_{3}}=\int_{\mathbb{R}^{2}} \mathrm{~d} y^{\prime} \int_{0}^{t} \frac{\partial^{2} B\left(x-y^{\prime}, \tau\right)}{\partial^{2} x_{3}} \frac{\partial v\left(y^{\prime}, t-\tau\right)}{\partial y_{1}} d \tau .
$$

Before we state our main Lemma, let us make some comments for boundary data $v\left(x^{\prime}, t\right)=g\left(\left|x^{\prime}\right|\right) h(t)$ in (5). Among arbitrary smooth functions satisfying certain properties (see [S] in page 4), we can choose an appropriate function $g$ such that for every point $x \in B_{x_{0}^{\prime}}^{+}$, the following condition holds:

$$
\int_{D_{1}}\left[\frac{1}{3\left|x^{\prime}-y^{\prime}\right|}\left(\frac{\partial g}{\partial y_{1}^{\prime}}\right)^{-}\left(\left|y^{\prime}\right|\right)-\frac{1}{\left|x^{\prime}-y^{\prime}\right|}\left(\frac{\partial g}{\partial y_{1}^{\prime}}\right)^{+}\left(\left|y^{\prime}\right|\right)\right] \mathrm{d} y^{\prime}>0,
$$


where $x^{\prime}$ is the projection of $x=\left(x^{\prime}, x_{3}\right) \in B_{x_{0}^{\prime}}^{+}$onto $\Lambda^{\prime}=B_{x_{0}^{\prime}} \cap\left\{x_{3}=0\right\}$. Indeed, since $g$ is radially symmetric and decreasing, the sign of $D_{y_{1}^{\prime}} g\left(\left|y^{\prime}\right|\right)$ is positive if $y_{1}<0$ and negative if $y_{1}>0$ in $D_{1}$. Due to the difference of the distance from $x^{\prime}$ to supports of $\left(\frac{\partial g}{\partial y_{1}^{\prime}}\right)^{-}$and $\left(\frac{\partial g}{\partial y_{1}^{\prime}}\right)^{+}$, (30) can be easily verified by an appropriate choice of $g$ satisfying [S]. Now we are ready to prove our main Lemma.

Main Lemma Let $v$ be a function given in (5), and assume that $g$ satisfies [S] and (30), and $h$ satisfies [T]. Let $u$ be given by the representation formula (12). Then for each $x \in B_{x_{0}^{\prime}}^{+}$, we have

$$
\left|\frac{\partial u_{i}(x, s)}{\partial x_{3}}\right| \geq C\left|\log \left(\frac{x_{3}}{2 \sqrt{s}}\right)\right|-C, \quad i=1,2,
$$

where $C=C\left(d\left(x_{0}^{\prime}, D_{1}\right), T\right)$.

The Proof of Main Lemma We only prove the case of $i=1$. Since it follows the same procedure line by line, we omit the details for the case of $i=2$. In addition, as mentioned earlier, it suffices only to consider $\left|\frac{\partial \tilde{u}_{2}(x, s)}{\partial x_{3}}\right|$, and therefore in this proof, we only prove the following estimate for $x \in B_{x_{0}^{\prime}}^{+}$and when $t=s$,

$$
\frac{\partial \tilde{u}_{2}(x, s)}{\partial x_{3}} \geq C\left|\log \left(\frac{x_{3}}{2 \sqrt{s}}\right)\right|-C .
$$

Simple computation shows that

$$
\frac{\partial^{2} B\left(x-y^{\prime}, \tau\right)}{\partial^{2} x_{3}}=e^{-\frac{x_{3}^{2}}{4 \tau}}\left(-\frac{1}{2 \tau}+\frac{x_{3}^{2}}{4 \tau^{2}}\right) \frac{1}{(4 \pi \tau)^{\frac{3}{2}}} K\left(x^{\prime}-y^{\prime}, \tau\right),
$$

where $B$ and $K$ are defined in (16) and (17), respectively. Thus, we obtain

$$
\begin{gathered}
\pi \frac{\partial \tilde{u}_{2}(x, s)}{\partial x_{3}}=\int_{\mathbb{R}^{2}} \mathrm{~d} y^{\prime} \int_{0}^{s} \frac{\partial^{2} B\left(x-y^{\prime}, \tau\right)}{\partial^{2} x_{3}} \frac{\partial v\left(y^{\prime}, s-\tau\right)}{\partial y_{1}} \mathrm{~d} \tau \\
=\frac{1}{(4 \pi \tau)^{\frac{3}{2}}} \int_{\mathbb{R}^{2}} \mathrm{~d} y^{\prime} \int_{0}^{s} e^{-\frac{x_{3}^{2}}{4 \tau}}\left(-\frac{1}{2 \tau}\right) \frac{1}{\tau^{\frac{3}{2}}} K\left(x^{\prime}-y^{\prime}, \tau\right) \frac{\partial v\left(y^{\prime}, s-\tau\right)}{\partial y_{1}} \mathrm{~d} \tau \\
+\frac{1}{(4 \pi \tau)^{\frac{3}{2}}} \int_{\mathbb{R}^{2}} \mathrm{~d} y^{\prime} \int_{0}^{s} e^{-\frac{x_{3}^{2}}{4 \tau}} \frac{x_{3}^{2}}{4 \tau^{2}} \frac{1}{\tau^{\frac{3}{2}}} K\left(x^{\prime}-y^{\prime}, \tau\right) \frac{\partial v\left(y^{\prime}, s-\tau\right)}{\partial y_{1}} \mathrm{~d} \tau \equiv I+J .
\end{gathered}
$$

We denote $d\left(x^{\prime}, y^{\prime}\right)=\left|x^{\prime}-y^{\prime}\right|$ by $\bar{d}_{y^{\prime}}$, for simplicity. We first show that the second term $J$ is bounded. Indeed, using the estimate (20) and $h(s-\tau)=\tau^{\frac{1}{2}}$, we have

$$
\begin{aligned}
J \leq & C\left\|\frac{\partial g\left(\left|y^{\prime}\right|\right)}{\partial y_{1}^{\prime}}\right\|_{\infty} \int_{D_{1}} \mathrm{~d} y^{\prime} \int_{0}^{s} e^{-\frac{x_{3}^{2}}{4 \tau}} \frac{x_{3}^{2}}{4 \tau^{2}} \frac{\tau^{\frac{1}{2}}}{\tau^{\frac{3}{2}}} \frac{\tau}{\bar{d}_{y^{\prime}}} \mathrm{d} \tau \\
& +C\left\|\frac{\partial g\left(\left|y^{\prime}\right|\right)}{\partial y_{1}^{\prime}}\right\|_{\infty} \int_{D_{1}} \mathrm{~d} y^{\prime} \int_{0}^{s} e^{-\frac{x_{3}^{2}}{4 \tau}} \frac{x_{3}^{2}}{4 \tau^{2}} \frac{\tau^{\frac{1}{2}}}{\tau^{\frac{3}{2}}}\left(\bar{d}_{y^{\prime}} e^{-C \frac{\vec{d}^{2}}{4 \tau}}\right) \mathrm{d} \tau \\
\equiv & C\left\|\frac{\partial g\left(\left|y^{\prime}\right|\right)}{\partial y_{1}^{\prime}}\right\|_{\infty}\left(J_{1}+J_{2}\right) .
\end{aligned}
$$


As before, we note that there exists a positive constant $C>0$ such that $C^{-1} d\left(x, y^{\prime}\right)<$ $\tilde{d} \equiv d\left(x_{0}^{\prime}, D_{1}\right)<C d\left(x, y^{\prime}\right)$ for all $x \in B_{x_{0}^{\prime}}^{+}$and $y^{\prime} \in D_{1}$. Now consider $J_{1}$.

$$
J_{1} \leq C \int_{0}^{s} e^{-\frac{x_{3}^{2}}{4 \tau}} \frac{x_{3}^{2}}{4 \tau} \frac{\tau^{\frac{1}{2}}}{\tau^{\frac{3}{2}}} \mathrm{~d} \tau \leq C \int_{\frac{x_{3}}{2 \sqrt{\tau}}}^{\infty} e^{-s^{2}} s \mathrm{~d} s \leq C
$$

Similarly, changing the variable $\frac{x_{3}^{2}+\tilde{d}^{2}}{4 \tau}=\rho^{2}$, we have

$$
J_{2} \leq C \int_{D_{1}} \mathrm{~d} y^{\prime} \int_{0}^{s} e^{-C \frac{x_{3}^{2}+\tilde{d}^{2}}{\tau}} \frac{x_{3}^{2}}{4 \tau^{3}} \mathrm{~d} \tau \leq C \int_{0}^{\infty} e^{-C \rho^{2}} \rho^{3} \mathrm{~d} \rho \leq C .
$$

Next consider the first term $I$.

$$
\begin{aligned}
I= & C \int_{\mathbb{R}^{2}} \mathrm{~d} y^{\prime} \int_{0}^{s} e^{-\frac{x_{3}^{2}}{4 \tau}}\left(\frac{1}{2 \tau}\right) \frac{1}{\tau^{\frac{3}{2}}} K\left(x^{\prime}-y^{\prime}, \tau\right)\left(\partial_{y_{1}} g\right)^{-}\left(\left|y^{\prime}\right|\right) h(s-\tau) \mathrm{d} \tau \\
& -C \int_{\mathbb{R}^{2}} \mathrm{~d} y^{\prime} \int_{0}^{s} e^{-\frac{x_{3}^{2}}{4 \tau}} \frac{1}{2 \tau} \frac{1}{\tau^{\frac{3}{2}}} K\left(x^{\prime}-y^{\prime}, \tau\right)\left(\partial_{y_{1}} g\right)^{+}\left(\left|y^{\prime}\right|\right) h(s-\tau) \mathrm{d} \tau \\
\equiv & I_{1}+I_{2} .
\end{aligned}
$$

With the estimate (21) of $K\left(x^{\prime}-y^{\prime}, \tau\right)$, we have

$$
\begin{aligned}
I_{1} \geq & C \int_{D_{1}} \mathrm{~d} y^{\prime} \int_{0}^{s} e^{-\frac{x_{3}^{2}}{4 \tau}}\left(\frac{1}{2 \tau}\right) \frac{1}{\tau^{\frac{3}{2}}} \frac{8 \pi \tau}{3 \bar{d}_{y^{\prime}}}\left(\partial_{y_{1}} g\right)^{-}\left(\left|y^{\prime}\right|\right) h(s-\tau) \mathrm{d} \tau \\
& -C \int_{D_{1}} \mathrm{~d} y^{\prime} \int_{0}^{s} e^{-\frac{x_{3}^{2}}{4 \tau}} \frac{1}{2 \tau} \frac{1}{\tau^{\frac{3}{2}}} \frac{8 \pi \tau}{3 \bar{d}_{y^{\prime}}}\left(e^{-C \frac{\bar{d}^{2}}{4 \tau}}\right)\left(\partial_{y_{1}} g\right)^{-}\left(\left|y^{\prime}\right|\right) h(s-\tau) \mathrm{d} \tau \\
\equiv & I_{11}+I_{12} .
\end{aligned}
$$

Let us show first that the second term $I_{12}$ is bounded. Indeed, using the facts that $\partial_{y_{1}} v=\left(\partial_{y_{1}} g\right)^{-} h$ is bounded and $\tilde{d}$ is strictly positive, we have

$$
\begin{aligned}
\left|I_{12}\right| & \leq C\left\|\partial_{y_{1}} v^{-}\right\|_{\infty} \int_{D_{1}} \mathrm{~d} y^{\prime} \int_{0}^{s} e^{-\frac{x_{3}^{2}}{4 \tau}} \frac{1}{\tau^{\frac{3}{2}}}\left(e^{\frac{-\tilde{d}^{2}}{4 \tau}}\right) \mathrm{d} \tau \\
& \leq C \frac{\left\|\partial_{y_{1}} v^{-}\right\|_{\infty}}{x_{3}^{2}+\tilde{d}^{2}} \int_{\frac{x_{3}^{2}+\tilde{\tilde{d}}^{2}}{2 \sqrt{s}}}^{\infty} e^{-\rho^{2}} \mathrm{~d} \rho \leq C
\end{aligned}
$$

where we used the change of variable $\frac{x_{3}^{2}+\tilde{d}^{2}}{4 \tau}=\rho^{2}$. Before estimating $I_{11}$, we consider first $I_{2}$. With the aid of upper bound (20) of $K\left(x^{\prime}-y^{\prime}, \tau\right)$, we get

$$
\begin{aligned}
I_{2} \geq & -C \int_{D_{1}} \mathrm{~d} y^{\prime} \int_{0}^{s} e^{-\frac{x_{3}^{2}}{4 \tau}} \frac{1}{2 \tau} \frac{1}{\tau^{\frac{3}{2}}} \frac{8 \pi \tau}{\bar{d}_{y^{\prime}}}\left(\partial_{y_{1}} g\right)^{+}\left(\left|y^{\prime}\right|\right) h(s-\tau) \mathrm{d} \tau \\
& -\int_{D_{1}} \mathrm{~d} y^{\prime} \int_{0}^{s} e^{-\frac{x_{3}^{2}}{4 \tau}} \frac{1}{2 \tau} \frac{1}{\tau^{\frac{3}{2}}}\left(\pi d_{x^{\prime}} e^{-C \frac{\bar{d}^{2}}{4 \tau}}\right)\left(\partial_{y_{1}} g\right)^{+}\left(\left|y^{\prime}\right|\right) h(s-\tau) \mathrm{d} \tau \\
= & I_{21}+I_{22} .
\end{aligned}
$$


It is easily checked that the second term $I_{22}$ is bounded by the similar calculation as (32). Indeed,

$$
\begin{aligned}
\left|I_{22}\right| & \leq C \int_{D_{1}} \mathrm{~d} y^{\prime} \int_{0}^{s} e^{-\frac{x_{3}^{2}}{4 \tau}} \frac{1}{2 \tau} \frac{1}{\tau^{\frac{3}{2}}}\left(\tilde{d} e^{-C \frac{\tilde{d}^{2}}{4 \tau}}\right)\left(\partial_{y_{1}} g\right)^{+}\left(\left|y^{\prime}\right|\right) h(s-\tau) \mathrm{d} \tau \\
& \leq C\left\|\left(\partial_{y_{1}} g\right)^{+}\right\|_{\infty} \int_{0}^{\infty} e^{-C \rho^{2}} \frac{\rho}{x_{3}^{2}+\tilde{d}^{2}} \mathrm{~d} \tau \leq C .
\end{aligned}
$$

To sum up all the above estimates, we obtain

$$
\begin{aligned}
\pi \frac{\partial \tilde{u}_{2}(x, s)}{\partial x_{3}} & =I+J \geq I-|J| \geq I_{1}+I_{2}-C \\
& \geq I_{11}+I_{21}-\left|I_{12}\right|-\left|I_{22}\right|-C \geq I_{11}+I_{21}-C .
\end{aligned}
$$

Thus it remains to estimate $I_{11}+I_{21}$. Since $g$ satisfies (30), we have

$$
\begin{aligned}
I_{11}+I_{21} & =C \int_{D_{1}} \mathrm{~d} y^{\prime} \int_{0}^{s} e^{-\frac{x_{3}^{2}}{4 \tau}} \frac{1}{\tau}\left(\frac{\left(\partial_{y_{1}} g\right)^{-}\left(\left|y^{\prime}\right|\right)}{3 \bar{d}_{y^{\prime}}}-\frac{\left(\partial_{y_{1}} g\right)^{+}\left(\left|y^{\prime}\right|\right)}{\bar{d}_{y^{\prime}}}\right) \mathrm{d} \tau \\
& =C \int_{D_{1}}\left(\frac{\left(\partial_{y_{1}} g\right)^{-}\left(\left|y^{\prime}\right|\right)}{3 \bar{d}_{y^{\prime}}}-\frac{\left(\partial_{y_{1}} g\right)^{+}\left(\left|y^{\prime}\right|\right)}{\bar{d}_{y^{\prime}}}\right) \mathrm{d} y^{\prime} \int_{\frac{x_{3}}{2 \sqrt{s}}}^{\infty} e^{-\rho^{2}} \frac{1}{\rho} \mathrm{d} \rho \\
& \geq C \int_{D_{1}}\left(\frac{\left(\partial_{y_{1}} g\right)^{-}\left(\left|y^{\prime}\right|\right)}{3 \bar{d}_{y^{\prime}}}-\frac{\left(\partial_{y_{1}} g\right)^{+}\left(\left|y^{\prime}\right|\right)}{\bar{d}_{y^{\prime}}}\right) \mathrm{d} y^{\prime}\left(-\log \left(\frac{x_{3}}{2 \sqrt{s}}\right)\right) .
\end{aligned}
$$

Summing up all together, we obtain

$$
\pi \frac{\partial \tilde{u}_{2}(x, s)}{\partial x_{3}} \geq I_{11}+I_{21}-C \geq C\left(-\log \left(\frac{x_{3}}{2 \sqrt{s}}\right)\right)-C .
$$

where $C=C\left(d\left(x_{0}^{\prime}, D_{1}\right), T\right)$. This completes the proof.

Remark 4.2 Calculations similar to those above gives that $u$ is Hölder continuous with the exponent $\alpha \in(0,1)$ in $\overline{B_{x_{0}^{\prime}}^{+}} \times I$, i.e.

$$
\left|u(x, t)-u\left(x^{\prime}, t^{\prime}\right)\right| \leq C\left(\left|x-x^{\prime}\right|+\left|t-t^{\prime}\right|^{\frac{1}{2}}\right)^{\alpha}
$$

where $C=C\left(d\left(x_{0}^{\prime}, D_{1}\right), T, \alpha\right)$. Since computations are similar to above Main Lemma and more or less straightforward, we omit the details.

Remark 4.3 If we take $h(s)=(s-t)^{\alpha}$ where $0<\alpha<\frac{1}{2}$ in (6), then we have

$$
\frac{\partial \tilde{u}_{2}(x, s)}{\partial x_{3}} \geq \frac{C}{x_{3}^{1-2 \alpha}}+C .
$$

Conversely, similar computation shows the reverse inequality, i.e.

$$
\frac{\partial \tilde{u}_{2}(x, s)}{\partial x_{3}} \leq \begin{cases}C \log \left(\frac{x_{3}}{2 \sqrt{s}}\right)+C & \text { when } \alpha=\frac{1}{2} \\ C x_{3}^{-1+2 \alpha}+C & \text { when } 0<\alpha<\frac{1}{2}\end{cases}
$$

where $C=C\left(d\left(x_{0}^{\prime}, D_{1}\right), T\right)$. 


\section{Weak derivative and pressure integrability}

Up to now, we have shown that the normal derivatives of tangential component $u$ blow up at the boundary $\Lambda^{\prime}=B_{x_{0}^{\prime}} \cap\left\{x_{3}=0\right\}$ at time $t=s$, which implies that $u$ can not be a classical solution. We will show that $u$ is a weak solution of the Stokes system (14) and (15). For convenience, we denote $Q_{x_{0}^{\prime}}^{+}=B_{x_{0}^{\prime}}^{+} \times I$ and $W_{\Lambda^{\prime}}^{1,2}\left(B_{x_{0}^{\prime}}^{+}\right)=\{u \in$ $W^{1,2}\left(B_{x_{0}^{\prime}}^{+}\right): u=0$ on $\left.\Lambda^{\prime}\right\}$. Here a weak solution $u$ means that $u \in L^{\infty}\left(I ; L^{2}\left(B_{x_{0}^{\prime}}^{+}\right)\right) \cap$ $L^{2}\left(I ; W_{\Lambda^{\prime}}^{1,2}\left(B_{x_{0}^{\prime}}^{+}\right)\right)$, div $u=0$ a.e. in $Q_{x_{0}^{\prime}}^{+}$, and

$$
\int_{Q_{x_{0}^{\prime}}^{+}}\left[u(x, t) v_{t}(x, t)-\nabla u(x, t) \nabla v(x, t)\right] \mathrm{d} x \mathrm{~d} t=0
$$

for every divergence free vector field $v \in \mathcal{C}_{0}^{\infty}\left(Q_{x_{0}^{\prime}}^{+}\right)$(see e.g. [14, page 171] for an analogous version of weak solutions). We note that once we have $u \in L^{2}\left(I ; W_{\Lambda^{\prime}}^{1,2}\left(B_{x_{0}^{\prime}}^{+}\right)\right)$, we easily obtain (33) by regularizing the function $h$ in (6) and passing to the limit. The pressure does not appear explicitly in this definition, but can be recovered modulo an arbitrary function of $t$. Formula (13) gives a representation of the pressure, and this representative is in $L^{q}\left(B_{x_{0}^{\prime}}^{+} \times I\right)$ for every $q<2$ (see Lemma 5.2). An equivalent approach would be to bring $p$ into the definition of weak solutions and replace (33) by

$$
\int_{Q_{x_{0}^{\prime}}^{+}}\left[u(x, t) v_{t}(x, t)-\nabla u(x, t) \nabla v(x, t)+p(x, t) \nabla \cdot v(x, t)\right] \mathrm{d} x \mathrm{~d} t=0
$$

for every $v \in \mathcal{C}_{0}^{\infty}\left(Q_{x_{0}^{\prime}}^{+}\right)$.

Lemma 5.1 Let $0<\epsilon<\frac{1}{2}$ and $v$ be a function given in (5), and assume that $g$ and $h$ satisfy $[\mathbf{S}]$ and $[\mathbf{T}]$, respectively. Let $u$ be given by the representation formula (12). Then $u \in L^{\infty}\left(I ; L^{2}\left(B_{x_{0}^{\prime}}^{+}\right)\right) \cap L^{2}\left(I ; W_{\Lambda^{\prime}}^{1,2}\left(B_{x_{0}^{\prime}}^{+}\right)\right)$and $\nabla u$ satisfies the following pointwise estimate in $B_{x_{0}^{\prime}}^{+} \times I$ :

$$
|\nabla u(x, t)| \leq\left\{\begin{array}{cl}
\frac{C}{(s-t)^{\frac{1}{2}-\epsilon}}+C \quad & \text { if } t<s \\
C|\log (t-s)|+C & \text { if } s<t<T,
\end{array}\right.
$$

where $C=C\left(d\left(x_{0}^{\prime}, D_{1}\right), T, \epsilon\right)$.

Proof. Note that $u \in L^{\infty}\left(I ; L^{2}\left(B_{x_{0}^{\prime}}^{+}\right)\right) \cap L^{2}\left(I ; W_{\Lambda}^{1,2}\left(B_{x_{0}^{\prime}}^{+}\right)\right)$is the easy consequence of estimate (34), Lemma 3.3, and Remark 4.2, and therefore we only show (34). In fact, it suffices to estimate $\partial_{x_{3}} u_{i}$ for $i=1,2$, because tangential derivatives are controlled in term of derivatives of $v$ and div $u=0$. In this proof, we consider only $\partial_{x_{3}} u_{1}$, because $\partial_{x_{3}} u_{2}$ follows similar computations. In addition, according to a priori estimates (28), we estimate only $\partial_{x_{3}} \tilde{u}_{2}$ in (29) because the other terms are controlled in terms of $L^{\infty}$ norm of $v$ and spatial derivatives of $v$.

Let us first consider the case $s<t<T$. For simplicity, we denote $\sup _{D_{1}}|D g|=$ $\|D g\|_{\infty}$ and as mentioned earlier, we note that there exists a positive constant $C>0$ 
such that $C^{-1} d\left(y, D_{1}\right)<\tilde{d} \equiv d\left(x_{0}^{\prime}, D_{1}\right)<C d\left(y, D_{1}\right)$ for all $y \in B_{x_{0}^{\prime}}^{+}$. Using the estimate (8) of $B(x, t)$ and $h(\tau)=0$ when $\tau>s$, we have

$$
\begin{aligned}
\left|\frac{\partial \tilde{u}_{2}(x, t)}{\partial x_{3}}\right| & \leq C\|D g\|_{\infty} \int_{D_{1}} \int_{0}^{s}\left|\frac{\partial^{2} B\left(x-y^{\prime}, t-\tau\right)}{\partial^{2} x_{3}}\right| h(\tau) \mathrm{d} \tau \\
& \leq C\|D g\|_{\infty} \int_{D_{1}} \int_{0}^{s} \frac{(s-\tau)^{\frac{1}{2}} \mathrm{~d} \tau}{\left(x_{3}^{2}+(t-\tau)\right)^{\frac{3}{2}}\left(\left(x-y^{\prime}\right)^{2}+(t-\tau)\right)^{\frac{1}{2}}} .
\end{aligned}
$$

Since $\left(\left(x-y^{\prime}\right)^{2}+(t-\tau)\right)>\bar{d}^{2} \equiv\left|x^{\prime}-y^{\prime}\right|^{2}>0$ and $(s-\tau) \leq(t-\tau)$, we have

$$
\begin{aligned}
\left|\frac{\partial \tilde{u}_{2}(x, t)}{\partial x_{3}}\right| & \leq \frac{C|| D g \|_{\infty}}{\bar{d}} \int_{0}^{s} \frac{(s-\tau)^{\frac{1}{2}} \mathrm{~d} \tau}{\left(x_{3}^{2}+(t-\tau)\right)^{\frac{3}{2}}} \leq \frac{C|| D g \|_{\infty}}{\bar{d}} \int_{0}^{s} \frac{\mathrm{d} \tau}{(t-\tau)} \\
& \leq \frac{C|| D g \|_{\infty}}{\tilde{d}}(|\log (t-s)|+|\log (s)|) \leq C|\log (t-s)|+C .
\end{aligned}
$$

In the case that $0<t<s$, we note first that

$$
\frac{\partial \tilde{u}_{2}(x, t)}{\partial x_{3}}=\int_{\mathbb{R}^{2}} \int_{0}^{t}\left(\partial_{t}-\sum_{i=1}^{2} \partial_{x_{i}^{2}}^{2}\right) B\left(x-y^{\prime}, t-\tau\right) \frac{\partial v\left(y^{\prime}, \tau\right)}{\partial y_{1}} \mathrm{~d} \tau
$$

where we used the fact that $B(x, t)$ satisfies the heat equation. Since $t$ is less than $s, v$ is smooth, and therefore using the integration by parts, we obtain

$$
\begin{gathered}
\frac{\partial \tilde{u}_{2}(x, t)}{\partial x_{3}}=\int_{\mathbb{R}^{2}} \int_{0}^{t} B\left(x-y^{\prime}, t-\tau\right) \partial_{\tau} \frac{\partial v\left(y^{\prime}, \tau\right)}{\partial y_{1}} \mathrm{~d} \tau \\
-\sum_{i=1}^{2} \int_{\mathbb{R}^{2}} \int_{0}^{t} B\left(x-y^{\prime}, t-\tau\right) \partial_{y_{i}^{2}}^{2} \frac{\partial v\left(y^{\prime}, \tau\right)}{\partial y_{1}} \mathrm{~d} \tau \equiv I+J,
\end{gathered}
$$

where we used the fact that $B(x, 0)=0$ and $v\left(y^{\prime}, 0\right)=0$. We first show that $J$ is uniformly bounded. Using the estimate (8) of $B(x, t)$, we obtain

$$
|J| \leq\left\|D^{3} g\right\|_{\infty} \int_{D_{1}} \int_{0}^{t} \frac{C(s-\tau)^{\frac{1}{2}} \mathrm{~d} y^{\prime} \mathrm{d} \tau}{\left(x_{3}^{2}+(t-\tau)\right)^{\frac{1}{2}}\left(\left(x-y^{\prime}\right)^{2}+(t-\tau)\right)^{\frac{1}{2}}} \leq \frac{C\left\|D^{3} g\right\|_{\infty}}{\tilde{d}},
$$

where we used the fact that $t-\tau \leq s-\tau$ and $\tilde{d}<C \bar{d}$. Thus, it remains to analyze the first term $I$. Using the estimate (8) again, we have

$$
\begin{aligned}
|I| & \leq C\|D g\|_{\infty} \int_{D_{1}} \mathrm{~d} y^{\prime} \int_{0}^{t} \frac{\left|\frac{\partial h(\tau)}{\partial \tau}\right| \mathrm{d} \tau}{\left(x_{3}^{2}+(t-\tau)\right)^{\frac{1}{2}}\left(\left(x-y^{\prime}\right)^{2}+(t-\tau)\right)^{\frac{1}{2}}} \\
& \leq \frac{C\|D g\|_{\infty}}{\bar{d}} \int_{0}^{t} \frac{1}{(s-\tau)^{\frac{1}{2}}\left(x_{3}^{2}+(t-\tau)\right)^{\frac{1}{2}}} \mathrm{~d} \tau \\
& \leq \frac{C\|D g\|_{\infty}}{\tilde{d}} \int_{0}^{t} \frac{1}{(s-\tau)^{\frac{1}{2}}(t-\tau)^{\frac{1}{2}}} \mathrm{~d} \tau .
\end{aligned}
$$


Since $(t-\tau)<(s-\tau)$, for any $\epsilon$ with $0<\epsilon<\frac{1}{2}$, we have

$$
\frac{1}{(s-\tau)^{\frac{1}{2}}(t-\tau)^{\frac{1}{2}}} \leq \frac{1}{(s-\tau)^{\frac{1}{2}-\epsilon}(t-\tau)^{\frac{1}{2}+\epsilon}} .
$$

With the aid of the inequality above, we get

$$
|I| \leq \frac{C|| D g \|_{\infty}}{\bar{d}(s-t)^{\frac{1}{2}-\epsilon}} \int_{0}^{t} \frac{1}{(t-\tau)^{\frac{1}{2}+\epsilon}} \mathrm{d} \tau \leq \frac{C\|D g\|_{\infty} t^{\frac{1}{2}-\epsilon}}{\tilde{d}(s-t)^{\frac{1}{2}-\epsilon}} \leq \frac{C}{(s-t)^{\frac{1}{2}-\epsilon}},
$$

where $C=C\left(d\left(x_{0}^{\prime}, D\right), T, \epsilon\right)$. To sum up estimates above, we obtain (34), which immediately implies that $\nabla u(x, t)$ is square integrable in $B_{x_{0}^{\prime}}^{+} \times I$. This completes the proof.

Next, we show that the pressure $p$ given in (13) is in the class $L^{q}\left(B_{x_{0}^{\prime}}^{+} \times I\right)$ for every $q<2$. Let us first recall the representation of the pressure already shown in (13).

$$
\begin{gathered}
p(x, t)=\frac{1}{2 \pi} \frac{\partial^{2}}{\partial^{2} x_{3}} \int_{\mathbb{R}^{2}} v\left(y^{\prime}, t\right) \frac{1}{\left|x-y^{\prime}\right|} \mathrm{d} y^{\prime} \\
-\frac{1}{\pi} \frac{\partial}{\partial x_{3}}\left(\frac{\partial}{\partial t}-\sum_{k=1}^{2} \frac{\partial^{2}}{\partial x_{k}^{2}}\right) \int_{\mathbb{R}^{2}} \mathrm{~d} y^{\prime} \int_{0}^{t} A\left(x-y^{\prime}, \tau\right) v\left(y^{\prime}, t-\tau\right) \mathrm{d} \tau \\
+\frac{1}{2 \pi} \int_{R^{2}} \frac{1}{\left|x-y^{\prime}\right|} \frac{\partial v\left(y^{\prime}, t\right)}{\partial t} \mathrm{~d} y^{\prime} \equiv I+J+K .
\end{gathered}
$$

Lemma 5.2 Let $v$ be a function given in (5), and assume that $g$ and $h$ satisfy [S] and $[\mathbf{T}]$, respectively. Let $p$ be given by the representation formula (13). Then $p \in$ $L^{q}\left(B_{x_{0}^{\prime}}^{+} \times I\right)$ for every $q<2$ and the following point-wise estimate holds in $B_{x_{0}^{\prime}}^{+} \times I$ :

$$
|p(x, t)| \leq\left\{\begin{array}{cl}
\frac{C}{(s-t)^{\frac{1}{2}}}+C & \text { if } t<s \\
C|\log (t-s)|+C & \text { if } s<t<t_{1}
\end{array}\right.
$$

where $C=C\left(d\left(x^{\prime}, D_{1}\right), T\right)$.

Proof. The first assertion is the easy consequence of (35). Thus it suffices to show the estimate (35). We note that there exists a positive constant $C>0$ such that $C^{-1} d\left(y, D_{1}\right)<\tilde{d} \equiv d\left(x_{0}^{\prime}, D_{1}\right)<C d\left(y, D_{1}\right)$ for all $y \in B_{x_{0}^{\prime}}^{+}$. The first term is uniformly bounded. Indeed,

$$
|I|=\frac{1}{2 \pi} \frac{\partial^{2}}{\partial^{2} x_{3}} \int_{\mathbb{R}^{2}} v\left(y^{\prime}, t\right) \frac{1}{\left|x-y^{\prime}\right|} \mathrm{d} y^{\prime} \leq \frac{C}{\tilde{d}^{3}} \sup _{B_{x_{0}^{\prime}}^{+} \times I}|v| .
$$

Next consider the third term $K$. If $t>s$, then $v\left(y^{\prime}, t\right)=0$, which implies $K=0$. Therefore it suffices to estimate it when $t \leq s$.

$$
|K| \leq C \int_{D_{1}} \frac{1}{\left|x-y^{\prime}\right|}\left|\frac{\partial v\left(y^{\prime}, t\right)}{\partial t}\right| \mathrm{d} y^{\prime} \leq \frac{C\|g\|_{\infty}}{\tilde{d}(s-t)^{\frac{1}{2}}} .
$$


It remains to estimate the second term $J$. For the second term, it suffices to estimate the part, denoted by $J$ again, containing the normal and time derivative because other terms containing normal and tangential derivatives can be easily shown to be bounded. First consider the case of $t<s$. using the estimate (7), we have

$$
\begin{aligned}
|J| & \leq C\|g\|_{\infty} \int_{D_{1}} \mathrm{~d} y^{\prime} \int_{0}^{t}\left|\frac{\partial_{\tau} h(\tau)}{(t-\tau)^{\frac{1}{2}}\left(\left(x-y^{\prime}\right)^{2}+(t-\tau)\right)}\right| \mathrm{d} \tau \\
& \leq \frac{C\|g\|_{\infty}}{\tilde{d}^{2}} \int_{0}^{t} \frac{1}{(s-\tau)^{\frac{1}{2}}(t-\tau)^{\frac{1}{2}}} \mathrm{~d} \tau \leq \frac{C\|g\|_{\infty} t}{\tilde{d}^{2}(s-t)^{\frac{1}{2}}} \leq \frac{C}{(s-t)^{\frac{1}{2}}} .
\end{aligned}
$$

In the case of $t \geq s$, using the estimate (7) again, we obtain

$$
\begin{aligned}
|J| & \leq C|| g \|_{\infty} \int_{D_{1}} \mathrm{~d} y^{\prime} \int_{0}^{s} \frac{(s-\tau)^{\frac{1}{2}}}{(t-\tau)^{\frac{3}{2}}\left(\left(x-y^{\prime}\right)^{2}+(t-\tau)\right)} \mathrm{d} \tau \\
& \leq C \frac{\|g\|_{\infty}}{\tilde{d}_{x^{\prime}}^{2}} \int_{0}^{s} \frac{(s-\tau)^{\frac{1}{2}}}{(t-\tau)^{\frac{3}{2}}} \mathrm{~d} \tau \leq C|\log (t-s)|,
\end{aligned}
$$

where we used $t-\tau \leq s-\tau$. To sum up above estimates, we obtain the point-wise estimate (35) of pressure, which immediately implies that $p \in L^{q}\left(B_{x_{0}^{\prime}} \times I\right)$ for every $q<2$. This completes the proof.

Summarizing the previous results, we obtain the following main theorem.

Main Theorem Let $v$ be a function given in (5), and assume that $g$ satisfies [S] and (30), and $h$ satisfies [T]. Let $u$ and $p$ be given by the representation formula (12) and (13), respectively. Then $(u, p)$ is a weak solution of the Stokes system (14) and (15). The pressure $p$ is in $L^{q}\left(B_{x_{0}^{\prime}}^{+} \times I\right)$ for any $q<2$ and $u$ is bounded and Hölder continuous up to the boundary in $\overline{B_{x_{0}^{\prime}, r}^{+}} \times I$ for any $0<r<1$, i.e. $u \in \mathcal{C}_{x, t}^{\alpha, \frac{\alpha}{2}}\left(\overline{B_{x_{0}^{\prime}, r}^{+}} \times I\right)$ where $0<\alpha<1$. The normal derivatives of tangential components of $u$ are unbounded at near $\Lambda^{\prime}=B_{x_{0}^{\prime}} \cap\left\{x_{3}=0\right\}$ when $t=s$.

The Proof of Main Theorem This is due to Lemma 3.3, Main Lemma, Remark 4.2, Lemma 5.1, and Lemma 5.2 above.

We conclude this section by constructing another example which is not even Hölder continuous up to the boundary.

Remark 5.3 We can also construct a weak solution, which is bounded but not Hölder continuous. For simplicity, let $s=1$ and $T=2$. We define $h(t)$ as follows

$$
h(t)=\left\{\begin{array}{cc}
\sum_{i=2}^{\infty} a_{i}(1-t)^{n_{i}} \xi(t) & \text { if } 0 \leq t \leq 1 \\
0 & \text { if } 1 \leq t \leq 2
\end{array}\right.
$$

where $a_{i}$ is a positive number satisfying $\sum_{i=2}^{\infty} i^{2} a_{i}<\infty$ and $n_{i}=\frac{1}{i}$, and $\xi$ is a cut-off function supported in $(0,2)$. Let $v$ be a function given in (5), and assume that $g$ satisfies [S] and (30), and h satisfies (37). Suppose $u$ be given by the representation formula (12). We note that since $h$ is bounded, so is $u$, i.e. $u \in L^{\infty}\left(B_{x_{0}^{\prime}}^{+} \times(0,2)\right)$ because of 
Lemma 3.3. Moreover, following the similar procedure as in Lemma 5.1, we obtain the following estimate of derivative of $u$ :

$$
|\nabla u(x, t)| \sim\left\{\begin{array}{cl}
C \sum_{i=2}^{\infty} \frac{i a_{i}}{(1-t)^{\frac{1}{2}-\frac{1}{2 i}}} & \text { if } t<1 \\
C a_{2}|\log (t-1)|+C \sum_{i=3}^{\infty} \frac{i a_{i}}{(t-1)^{\frac{1}{2}-\frac{1}{2 i}}} & \text { if } 1<t<2 .
\end{array}\right.
$$

This implies $|\nabla u(x, t)| \in L^{2}\left(B_{x_{0}^{\prime}}^{+} \times(0,2)\right)$. Indeed, simple calculations show

$$
\begin{gathered}
\int_{0}^{2} \int_{B_{x_{0}^{\prime}}^{+}}|\nabla u|^{2} \leq C \int_{0}^{1}\left(\sum_{i=2}^{\infty} \frac{i a_{i}}{(1-\tau)^{\frac{1}{2}-\frac{1}{2 i}}}\right)^{2} \mathrm{~d} \tau \\
+C \int_{1}^{2}\left(a_{2}|\log (\tau-1)|+\sum_{i=3}^{\infty}+\frac{i a_{i}}{(1-\tau)^{\frac{1}{2}-\frac{1}{2 i}}}\right)^{2} \mathrm{~d} \tau \\
\leq C a_{2}^{2}+C\left(\sum_{i=2}^{\infty} i a_{i}\right) \int_{0}^{1} \sum_{i=2}^{\infty} \frac{i a_{i}}{(1-\tau)^{1-\frac{1}{i}}} \mathrm{~d} \tau \\
\leq C a_{2}^{2}+C\left(\sum_{i=2}^{\infty} i a_{i}\right)\left(\sum_{i=2}^{\infty} i^{2} a_{i}\right)<\infty .
\end{gathered}
$$

where we used $\sum_{i=2}^{\infty} i a_{i} \leq C \sum_{i=2}^{\infty} i^{2} a_{i}$. On the other hand, we note that

$$
\left|u\left(x^{\prime}, x_{3}, 1\right)\right| \geq C a_{i} x_{3}^{\frac{2}{i}} \int_{\frac{x_{3}}{2}}^{\infty} e^{-\rho^{2}} \rho^{-\frac{2}{2}} d \rho \quad \text { for all } i \geq 2 .
$$

From this observation, we note that $u$ is not Hölder continuous up to the boundary. Indeed, for a fixed $h>0$, since $i$ can be chosen to be arbitrary large, we have $\left|u\left(x^{\prime}, x_{3}, 1\right)\right| x_{3}^{-h} \geq C x_{3}^{\frac{2}{i}-h}$. We can see that the right-side goes to infinity as $x_{3}$ goes to zero provided that $\frac{2}{i}-h$ is negative, and therefore it cannot be Hölder continuous. Next, by following similar procedures as in Lemma 5.2, we can see that the pressure $p$ represented in (13) satisfies the following point-wise estimate:

$$
|p(x, t)| \sim \begin{cases}C \sum_{i=2}^{\infty} \frac{i a_{i}}{(1-t)^{1-\frac{1}{i}}} & \text { if } t<1 \\ C a_{2}|\log (t-1)|+C \sum_{i=3}^{\infty} \frac{i a_{i}}{(t-1)^{\frac{1}{2}-\frac{1}{2 i}}} & \text { if } 1<t<2 .\end{cases}
$$

It can be easily checked that $p \in L^{1}\left(B_{x_{0}^{\prime}}^{+} \times(0,2)\right)$, but it is not in $L^{q}\left(B_{x_{0}^{\prime}}^{+} \times(0,2)\right)$ for any $q>1$. This shows that some assumptions on $p$ are necessary to get Hölder continuity up to the boundary. We remark that if $p \in L^{q}\left(B_{x_{0}^{\prime}}^{+} \times(0,2)\right), q>1$, then $u$ is Hölder continuous with the exponent $\alpha=\alpha(q)$ (see Lemma 1 and Lemma 2 in [9, page 216, 219]). 


\section{ACKNOWLEDGEMENT}

This research was supported in part by NSF Grant No. DMS-9877055. The author express his deep gratitude to his advisor, Professor Vladmír Šverák for guidance and encouragement.

\section{References}

[1] S. Agmon, A. Douglis \& L. NiRenberg Estimates near the boundary for solutions of elliptic partial differential equations satisfying general boundary conditions. II, Comm. Pure Appl. Math. 17 (1964), 35-92.

[2] L. CAffarelli, R. Kohn \& L. NiRenberg Partial regularity of suitable weak solutions of the Navier-Stokes equations, Comm. Pure Appl. Math. 35 (1982), 771831.

[3] G. P. GALDI An introduction to the mathematical theory of the Navier-Stokes equations. Vol I \& II, Springer-Verlag, New York, 1994.

[4] D. Gilbarg \& N. S. TRUDINGER Elliptic partial differential equations of second order, Springer-Verlag, Berlin, 2001.

[5] E. Hopf Über die Anfangswertaufgabe für die hydrodynamischen Grundgleichungen (German), Math. Nachr. 4, (1951), 213-231.

[6] O. A. LADYZhenskaya The mathematical theory of viscous incomprehensible flow, Second English edition, Gordon and Breach, Science Publishers, New YorkLondon-Paris 1969.

[7] O. A. Ladyzhenskaya, V. A. Solonnikov \& N. N. Uralceva Linear and Quasilinear Equations of Parabolic type, Translations of Mathematical Monographs, 23, Amer. Math. Soc., Providence, R.I., 1968.

[8] J. LERAY Sur le mouvement d'un liquide visqueux emplissant l'espace, Acta. Math. 63 (1934), 193-248.

[9] G. A. SEREgin Some estimates near the boundary for solutions to the nonstationary linearized Navier-Stokes equations, Zap. Nauchn. Sem. S.-Peterburg. Otdel. Mat. Inst. Steklov. (POMI), 271, (2000), pp 204-223.

[10] J. SERRIN On the interior regularity of weak solutions of the Navier-Stokes equations, Arch. Rational. Mech. Anal. 9 (1962), 187-195.

[11] H. SOHR \& W. VON WAHL On the regularity of pressure of weak solutions of the Navier-Stokes equations, Arch. Math. 46 (1986), 428-439.

[12] V. A. SOLONNIKov Estimates of solutions of nonstationary linearized systems of Navier-Stokes equations, Trudy Mat. Inst. Steklov 70 (1964), 213-317. (in English:A.M.S. Translations, Series II 75, 1-117). 
[13] E. M. STEIN Singular integrals and differentiability properties of functions, Princeton Univ. Press, Princeton, N.J., 1971

[14] R. TEMAN Navier-Stokes equations. Theory and numerical analysis, Reprint of the 1984 edition. AMS Chelsea Publishing, Providence, RI, 2001

\section{KYUNGKEUN KANG}

School of Mathematics

University of Minnesota

Minneapolis, MN 55455

USA

E-mail : kkang@math.umn.edu

KEY WORDS AND PHRASES : Stokes system, boundary regularity.

2000 MATHEMATICS SUBJECT CLASSIFICATION : 35Q30, 76D03, 76 D07. 